\title{
The Realization of Digital Vector Controlled SVPWM Frequency-Variable Speed-Adjustable System
}

\author{
SHE Yan \\ Shunde Polytechnic, Foshan 528300, China \\ Shelley186@sohu.com
}

Keywords: FOC; SVPWM; DSP; Frequency-Variable Speed-Adjustable System

\begin{abstract}
The paper introduces the field oriented control (FOC) and voltage space vector pulse wide modulation (SVPWM) arithmetic, develops a suit of Digital Vector Controlled frequency -variable speed-adjustable system based on digital signal processor (DSP) TMS320LF2407 of TI company, and research and analyze holistic design project of the system, presents the instantiation application result. The experimental results indicate: the laboratorial setting has a simpler hardware construction, higher control precision, less output current harmonics distortion and better real-time signal processing.
\end{abstract}

\section{Introduction}

With the development of control theory and the AC drive theory, frequency-variable speed-adjustable technology also has been rapid progress. The development and applications of Pulse width modulation (PWM) technology has promoted frequency conversion device for high performance, it has played a major role and popularity for the application and popularization of speed control technology. Voltage space vector pulse width modulation (SVPWM) technology overcome all the disadvantages of the phase control principle, the AC motor stator has been close to the sine voltage and current wave, to improve the efficiency of the motor output. Most modern PWM generation circuit has used a high-speed output of the microcontroller and digital signal processor (DSP), generated PWM by software programming[1].The TMS320LF2407 DSP controller peripheral functions and real-time processing capabilities rolled into one, with a better processing performance (30MIPS), larger program memory, more integrated peripherals, faster A/D conversion and More useful digital control of the motor[2]. This article describes the basic principle of SVPWM control and the system design plan, to achieve all-digital frequency-variable speed-adjustable system with TMS320LF2407 as the master chip. The experimental results show that the use of the control system has simple hardware circuit, software development easier, the control precision and reliability higher, real-time stronger and other advantages.

\section{Vector control}

The idea of vector control is asynchronous motor simulation into dc motor to control. Through coordinate transformation, the stator current vector is decomposed into the two dc component of rotor magnetic field orientation, and the control respectively, so as to realize flux and torque decoupling control, to achieve the dc motor control effect. After the corresponding coordinate transformation, will be able to control the asynchronous motor.

\section{Design of Hardware Structure}

The whole system main circuit adopts AC-DC-AC voltage source type inverter structure, mainly composed by the AC / DC rectifier and filter circuit, an inverter, detection and control circuit. The system is a closed-loop speed feedback control system, DSP is responsible for sampling the phase current, voltage, calculating the motor speed and position based on photoelectric encoder data, the control signal through the optically coupled isolation circuit, controlled the power switch device 
on-off of inverter circuit, generating a three-phase AC signal to drive an AC motor running. At the same time, through the voltage, current detection, DSP control running state of variable frequency speed control system, when the system short circuit, over-current, over-voltage fault, DSP will blockade the PWM signal, and make the motor stops rotating, and displayed by the indicator, as shown in Figure 1[3].

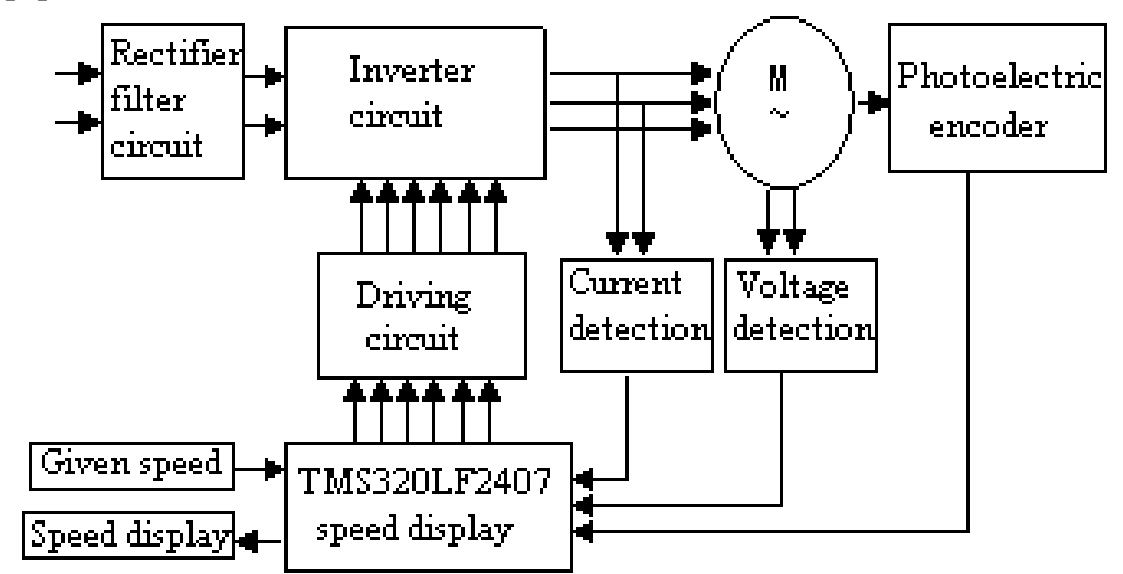

Fig. 1 Schematic diagram of frequency conversion speed control device

\section{The control circuit design}

The design of control circuit plays a crucial role in the United States to the variable frequency drive system, TMS320LF2407 introduced by The United States TI (Texas Instruments)applied to industrial control,the low-cost, high-performance DSP cores and some advanced peripherals was integrated into a chip, especially suited to digital motor control applications, the DSP is a high-speed dedicated microprocessors to provide advanced, reliable and efficient signal treatment and control for high-performance transmission control. Taking TMS320LF2407 as the core control circuit diagram include DSP small system circuit, current and speed detecting circuit, the PWM output, RS485/RS232 communication interface circuit ,and so on[4].

\section{A small DSP system circuit}

DSP is a dedicated microprocessor, By static CMOS technology with high performance, TMS320LF2407 make the supply voltage down to 3.3V, Contain CPU with operating rate of 30MIPS (million instructions per second); The DSP integrated perfect memory and peripherals: up to $1.5 \mathrm{~K}$ word-chip data/program RAM, up to $32 \mathrm{~K}$ words flash program memory, 544 word dual-port RAM and $2 \mathrm{~K}$ words single-port RAM, expandable external memory total 192K word space; based on phase-locked loop (PLL) clock module, watch dog timer (WDT), synchronous serial peripheral interface (SPI), asynchronous serial communication interface (SCI), controller area network (CAN) ; dual 10-bit ADC with 16 analog signals, one A/D converter the minimum time 500ns; 40 individually programmed or re-used general-purpose I/O pins; 5 external interrupt; The DSP use simulation technology based on JTAG (an international standard test protocol) scan; for pulse width modulation (PWM) control of the two event manager, each contains two general-purpose timers, 3 compare and capture unit, 2 encoding unit with the optical encoder interface, 8 PWM outputs, the PWM waveform production unit contains programmable dead time control, export the symmetric and asymmetric PWM or space vector PWM waveforms[5].

As TMS320LF2407 simulation execution needs the external program memory, the system uses 16-bit up to 12ns speed external program memory CY7C1021. With reset circuit, crystal oscillator-based PLL clock input and JTAG emulation interface, compose the DSP small system circuit. 


\section{Detection circuit design and implementation [6]}

Detection circuit is to detect all kinds signals, converted into the digital signal, which can be identified by DSP. Detecting circuit consists of a current detection module and the motor speed and position detection module.

These test signals are sent to directly to the TMS320LF2407 internal interface through the peripheral interface circuit, then according to the corresponding set, read the detected specific numerical.

\section{PWM output and fault input circuit}

Through the DSP programming, produces 6-channel programmable dead and variable output polarity of the PWM waveform, drive IPM inverter module power device of the main circuit,through a drive circuit and a photoelectric isolation circuit, obtain sine waveform of a variable-voltage and variable-frequency effect, supply electrical power. Circuit shown in figure 2.

PWM signal output request high speed isolation, otherwise it will cause the serious consequences of a short circuit. The fault input signal must be sent to the main control board after the isolation.

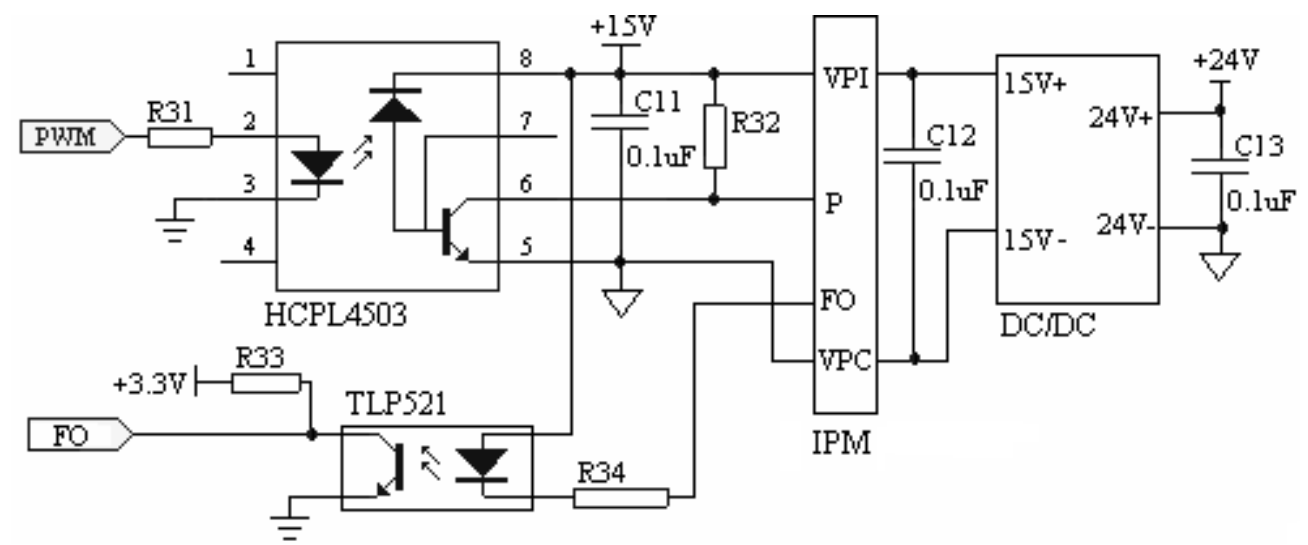

Fig.2 PWM isolation output IPM circuit

\section{The System Software Design}

The system software main program complete the main program main features of the initialization of the DSP, parameter settings and real-time display of the speed. SVPWM interrupt service subprogram mainly complete the sample of the current value, the calculation of the current speed and $\theta$, the vector transformation, and SVPWM waveform output.

Main program is divided into two: the system initialization and the variable initialization. the system initialization refers to the initialization of before DSP running, including: set the system clock; allow the serial receive interrupt; initialize a variety of I /O port; initialize AD sample; set Event Manager module, 6-phase PWM output, set the dead time, enable quadrature encoder pulse circuit. The variable initialization means that the initial assignment of the storage unit corresponding to the variables[7]. specifically main program and SVPWM interrupt service subprogram flow shown in Figure 3(a).

SVPWM interrupt service subprogram task mainly: (1) be responsible for the current sampling of A, B two-phase, and the realization of A/D conversion, (2) calculate the current value of speed and $\theta$ according to the message of the pulse encoder, (3) complete transformation and calculation of the vector according to the measured value, (4) output SVPWM. SVPWM interrupt service subprogram flow shown in Figure 3(b). 


\section{Conclusions}

In order to avoid harmonic frequency loss and pollution of the experimental device, SVPWM with high DC voltage efficiency will replace the traditional SPWM wave. The frequency-variable speed-adjustable system is with DSP LF2407 chip at the core, can improve the system response speed. Set motor parameters: Rated power $750 \mathrm{~W}$, rated voltage $380 \mathrm{~V}$, rated current $1.7 \mathrm{~A}$, rated speed $2850 \mathrm{r} / \mathrm{min}$. Inverter switching frequency is $2.4 \mathrm{kHz}$, speed is given as $314 \mathrm{rad} / \mathrm{s}$. According to the experiment on the experimental device, the measured inverter output 50HZ SVPWM waveforms, the experimental results show that the use of DSP and SVPWM, can make output voltage harmonic less distortion, DC voltage more efficient. The system can quickly reach the steady state almost no overshoot, show that the system has good dynamic and static performance.

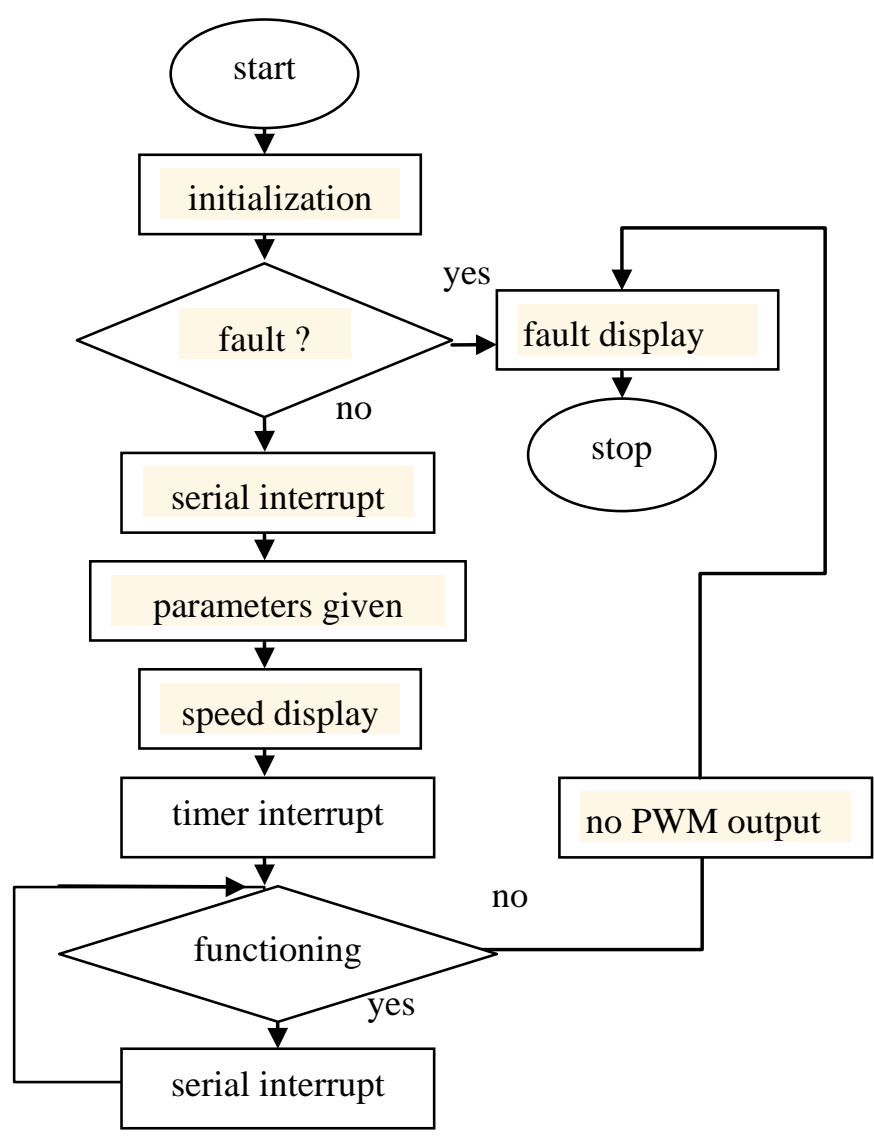

(a)

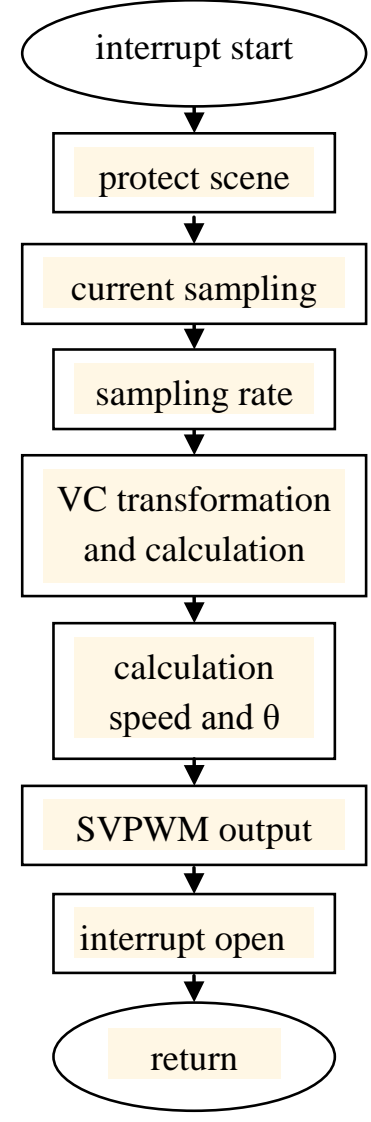

(b)

Figure 3 the main program and SVPWM interrupt service subprogram

\section{Reference}

[1] Li Huade. Alternating Current Speed-Adjustable System[M]. Beijing: Press of Electronics Industry, 2003.3.

[2] Liu Heping, Yan Lifeng, Zhang Xuefeng. TMS320LF240xDSP Structure Principle and Application[M]. Beijing: Beijing University of Aviation and Avigation Press, 2002.9.

[3] Wang Chenyuan, Li Haodong, Xia Jiakuan. Study of Intelligent Vector Control System Based on TMS320F240[J]. Applied Power Electronics Conference and Exposition, 2002, 2 (5): 550-553.

[4] Wu shouzhen, Zang Yingjie. SPWM Technology Electrical Transmission[M], 2003.1.

[5] Li Yong, Huang Lipei, li Fahai. All Digital Space Vector PWM Induction Motor Drive Based on Flux Control[J]. IEEE TENCON, 2003, 3 (8): 598-601. 
[6] Wang Xiaoming. Single chip Control of Motor[M]. Beijing: Beijing University of Aviation and Avigation Press, 2002.5.

[7] Wang Xiaoming, Wang Ling. DSP Control of Motor[M]. Beijing: Beijing University of Aviation and Avigation Press, 2004.7. 